\title{
RELIABLE TIMING SYSTEMS FOR COMPUTER CONTROLLED ACCELERATORS
}

\author{
Jürgen KNOTT and Robert NETTLETON \\ CERN, Geneva, Swizerland
}

Over the past decade the use of computers has set new standards for control systems of accelerators with ever increasing complexity coupled with strngent reliability criteria. In fact, with very slow cycling machines or storage rings any erratic operation or timing pulse will cause the loss of precious particles and waste hours of time and effort of preparation. Thus, for the CERN linac and LEAR (Low Energy Antiproton Ring) timing system reliability becomes a crucial factor in the sense that all components must operate practically without fault for very long periods compared to the effective machine cycle. This has been acheved by careful selection of components and design well below thermal and electrical limits, using error detection and correction where possible, as well as developing "safe" decoding techniques for serial data trains. Further, consistent structuring had to be applied in order to obtain simple and flexible modular configurations with very few components on critical paths and to minimize the exchange of information to synchronize accelerators. In addition, this structuring allows the development of efficient strategies for on-line and off-line fault diagnostics. As a result, the timing system for Linac 2 has, so far, been operating without fault for three years, the one for LEAR more than one year since its final debugging.

\section{Introduction}

We report a simple design concept for large computer controlled timing systems, as applied to two basically very different machines of the CERN accelerator complex:

1) the two $50 \mathrm{MeV}$ linacs used as pulsed injectors (ca. 1 $s$ repetition) for protons and other hadronic particles to the PSB-PS-SPS series of accelerators;

2) the LEAR accelerator/storage ring, which operates independently for periods of hours after injection of either antiprotons from the PS or protons or $\mathrm{H}^{-}$for the purpose of machine tests from Linac 1.

Operation and sharing of common beam transfer lines (fig. 1) requires the controls system of these machines to be synchronized with that of the proton synchrotron (PS), which, in turn, has to serve its various users with beams of quite different characteristics.

The challenge was to get manageable systems by

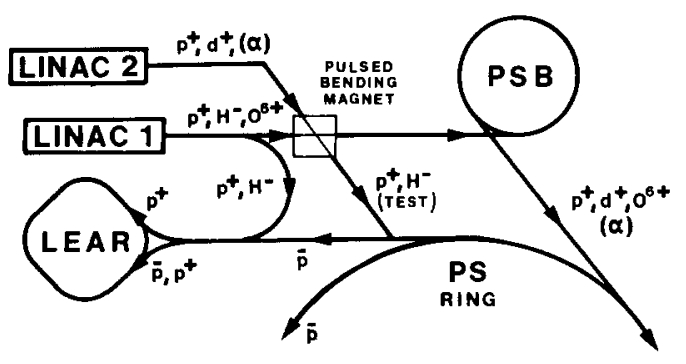

Fig. 1. Transfer lines between the linacs, LEAR and the PS/PSB. using a small number of reliable modules and to keep the operational aspect simple.

\section{Structured design}

The division into clear modular blocks permits a complex system to appear simple instead of complicated. It allows the exchange of reference pulses, as required to synchronize successive accelerators, to be minimized and to simplify the protocols for pulse and beam transfers.

The simple structure, as used in our three systems, permits one to get easily familiarized with it (fig. 2). The actual user timing is generated with the specific timing modules (delays, logic gates, etc.) "hooked" onto an appropriate reference pulse and cycle information, if required. Extension of the system is done by just adding modules to the corresponding prepulses and linking them to the computer control system. Each particular user output is, thus, clearly defined by the input conditions (reference) and the control value (fig. 3 ).

The basic operation relies on a few essential units on a restricted critical path, separated from those for secondary tasks like data acquisition. Nesting and side links between modules should, if possible, be avoided.

\subsection{Practical layout}

The three systems are mainly built from seven common standardized modules and a few dedicated units designed for the specific needs of each machine. Their number represents between $5 \%$ and $10 \%$ of the total control equipment. 


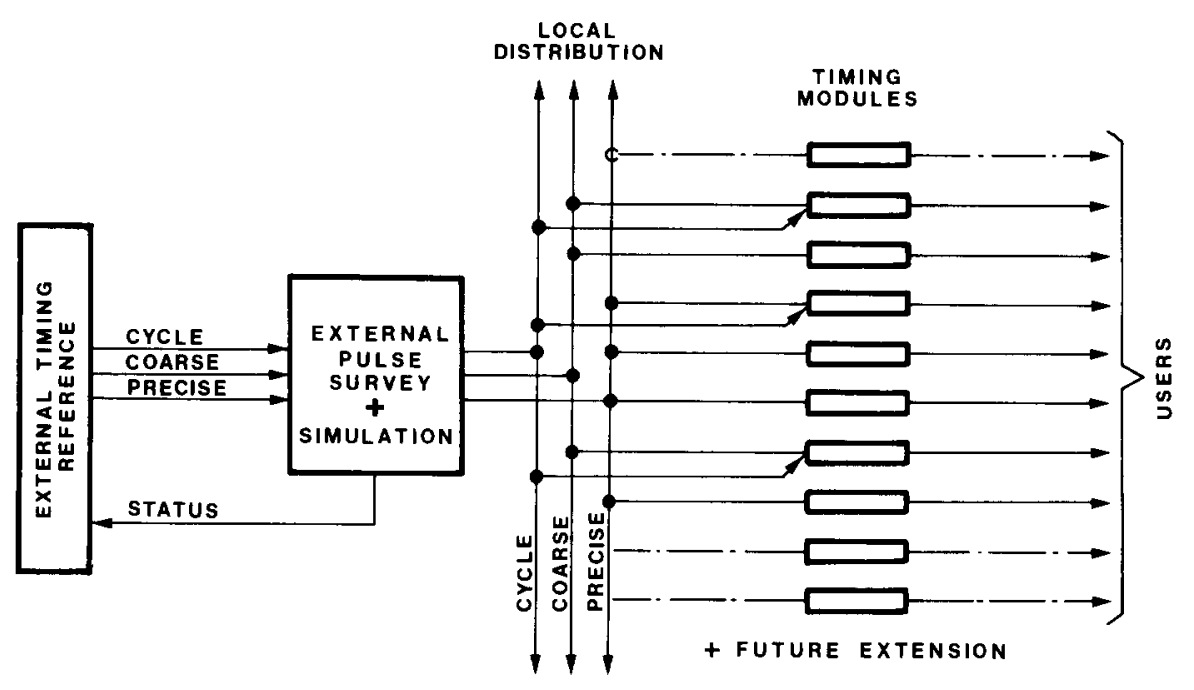

Fig. 2. Structure of timing systems.

All the timing units are distributed among the local controls, but kept together in clusters for the common sharing of facilities like clock generators for the digital delays. Those requiring frequent access by the computer system are mostly housed in CAMAC crates, linked by serial CAMAC to the process computer (DEC LSI 11/73) [1]. Simpler logic modules are built to the NIM standard.

\section{Reference pulses}

The various machines in a chain of accelerators need to exchange two groups of reference signals for the basic operation:

1) coarse timing information for controlling slow equipment and perhaps advance cycle information, if several users with different requirements are to be served on successive cycles;

2) precise timing related to the energy of beam transfer involving a circular machine and, if applicable, $r f$ phased signals for bunch synchronization.

The timing systems described use a coded "supercycle" information, distributed by the PS control system

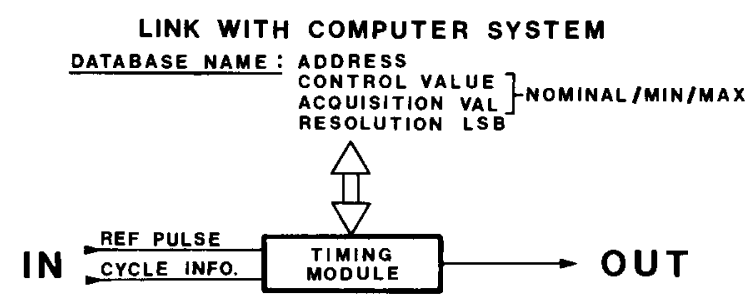

Fig. 3. Definition of a user parameter. at the repetition rate of the linacs. In addition, the linacs receive two and LEAR three prepulses from this system.

In future, the information of both groups could be combined, for example by coding them on the rising and falling edges of the same pulse. Such a simplification would make checking the rate of occurrence and correct sequence of the incoming reference pulses much easier. This kind of surveillance has already proved very successful for detecting faults during transmission or on preceding stages of the timing system.

Errors may be corrected where possible, such as by substituting missing or badly timed pulses. In most cases this cannot restore full operation and will most likely impede further acceleration of particles. But it will at least protect sensitive apparatus at the users level and allow for an automatic restart of operation after the fault has been cured.

\section{System performance}

The physical reliability is determined by the fault rate of the equipment. It is influenced by designing to reasonable electrical and thermal limits, as well as providing protection against electromagnetic interference.

As an example, reducing by $20 \%$ the output voltage of level adapters, as used for long distance pulse transmission, has increased their lifetime from a few breakdowns per year a decade ago to several years of fault-free operation. Similar results have been obtained by limiting the power consumption of CAMAC equipment to 3 W per slot. For already existing modules this has been partially achieved by retro-fitting or redesigning with modern low power components. 
The operational reliability is more difficult to quantify as it accounts for the time the system experts and operators have to spend on fault location. It is mainly influenced by:

- The complexity of the system and its interconnection with dependent systems;

- the efficiency of diagnostic facilities, e.g. synoptic displays and test programs available on the computer system;

- a convenient presentation of the system to the operators;

- and to some extent on the fault tolerance of the system, as obtained via automatic survey of transmission protocols in critical places.

\section{LEAR event timing}

Particular precautions had to be taken for the distributed event timing for LEAR. In fact, a dedicated timing event generator takes over the control of the machine cycle for periods up to $24 \mathrm{~h}$ after injection of particles [2,3].

A total of 256 events may be programmed with a resolution of $1 \mathrm{~ms}$. This information is distributed on a serial train to the equipment around the machine, mainly to control the sequence of about 50 autonomous function generators for the magnetic lattice and acceleration.

This system must be extremely reliable, as any erratic event can cause the different generators to get out of step and, consequently, bring about the loss of the scarce antiprotons.

For this purpose the events are decoded locally by using quite narrow data windows, controlled and rephased continuously by a quartz oscillator logic. Each event has to be enabled and routed individually to one of the eight outputs of each decoder by an additional software code.

With these precautions and very precise event train repeaters, we presently obtain error rates of better than $10^{-11}$ or practically fault-free operation over several weeks.

\section{Conclusion}

With the present availability of our systems for very long periods we consider the technological aspects of large timing systems to have been mastered.

However, despite the little attendance they require, we must admit that we are from time to time faced with new problems which have their origin in the constant evolution of the complexity of operation and the addition of new facilities. These problems are enhanced by the fact that most of our accelerators have, for historical reasons, different control concepts. Consequently, we continuously need to upgrade our diagnostic tools in order to adapt to these new situations.

\section{References}

[1] A. Cheretakıs, J. Knott, P. Mead, A. van der Schueren and U. Tallgren, The Control System of the CERN New Linac, Proc. 1976 Proton Linear Accelerator Conf., Chalk River, Ontario, Atomic Energy of Canada Ltd., AECL-5677 (1976).

[2] U. Tallgren, D. Kemp, J. Knott, P. Liénard, T. Pettersson and A. van der Schueren, The Distributed Control System for the CERN LEAR, 6th IFAC Workshop, Monterey (1985) CERN PS/85-27 (LI).

[3] J. Knott, The LEAR Control Timing System, CERN PS/LI/Note 83-12. 